Published in "Journal of Global Antimicrobial Resistance 13: 37-39, 2018"

which should be cited to refer to this work.

\section{Colistin-resistant carbapenemase-producing isolates among Klebsiella spp. and Acinetobacter baumannii in Tripoli, Libya}

Sir,

The emergence of acquired carbapenemases is a serious threat to public health worldwide, forcing the use of last-resort antibiotics such as polymyxins. Use of such molecules had recently led to the emergence of colistin-resistant carbapenemase-producing isolates, leaving only a few therapeutic options for the near future. Here we report the identification of colistin-resistant isolates among a collection carbapenemase-producing Enterobacteriaceae and Acinetobacter baumannii isolates.

A total of 61 imipenem-non-susceptible isolates were recovered from different clinical samples from patients in Tripoli Medical Centre (Tripoli, Libya) between 2014 and 2015. Among this collection, 32/61 were identified as Klebsiella pneumoniae, 28/61 as $A$. baumannii and $1 / 61$ as Klebsiella oxytoca using API ${ }^{\circledR}$ gallery (bioMérieux, Marcy-l'Étoile, France).

Antimicrobial susceptibility testing was performed according to Clinical and Laboratory Standards Institute (CLSI) recommendations. Carbapenemase activity was determined using the RAPIDEC $^{\circledR}$ CARBA NP test (bioMérieux) for Enterobacteriaceae and the modified CarbAcineto NP test for A. baumannii, showing positive results for all isolates. Resistance to colistin was determined with the Rapid Polymyxin ${ }^{\mathrm{TM}}$ NP test for Enterobacteriaceae [1]. Imipenem and colistin minimum inhibitory concentrations (MIC) were evaluated by Etest and broth microdilution, respectively. All isolates presenting a colistin MIC $>2 \mu \mathrm{g} / \mathrm{mL}$ were considered resistant. All data are summarised in Table 1.

The resistance phenotype towards other families of antibiotics was determined using the standard disk diffusion method. All of the isolates presented a multidrug-resistant phenotype. Molecular investigations involved the detection of carbapenemases, 16S rRNA methylases and colistin resistancerelated genes by PCR amplification using specific primers, followed by sequencing (Microsynth AG, Balgach, Switzerland). the $28 \mathrm{~A}$. baumannii isolates were positive for the $b l a_{\mathrm{OXA}-23}$ gene and $12 / 28$ were positive for the armA 16S rRNA methylaseencoding gene conferring high-level resistance to aminoglycosides. In addition, 5/28 isolates were resistant to colistin with an MIC between $4 \mu \mathrm{g} / \mathrm{mL}$ and $128 \mu \mathrm{g} / \mathrm{mL}$ with an unknown resistance mechanism.

Among the 33 carbapenem-resistant Klebsiella spp. isolates, 28/ 33 and 20/33 were positive by PCR for the bla $a_{\mathrm{OXA}-48}$ and $b l a_{\mathrm{NDM}-1}$ genes, respectively. Noteworthy, 15/33 isolates were positive for both carbapenemase genes. Six isolates were found to be resistant to colistin with an MIC ranging between $64 \mu \mathrm{g} / \mathrm{mL}$ and $128 \mu \mathrm{g} / \mathrm{mL}$ (Table 1). Noteworthy, the 16S rRNA methylase-encoding gene rmtC was detected in three $K$. pneumoniae isolates and one $K$. oxytoca isolate.

The clonal relationship of the Klebsiella and Acinetobacter isolates was evaluated by pulsed-field gel electrophoresis (PFGE). Briefly, total DNA from bacterial isolates was digested by XbaI or Apal restriction enzymes (New England Biolabs, Ipswich, MA) for the Klebsiella and Acinetobacter isolates, respectively. The generated fragments were separated using a CHEF-DR ${ }^{\circledR}$ III System (BioRad) and the different pulsotypes were identified using the MultiVariate Statistics Package (MVSP) software. All PFGE profiles showing a similarity coefficient $>0.8$ were assigned to the same cluster. Multilocus sequence typing (MLST) was performed for the colistin-resistant isolates and sequence types (STs) were assigned using the online databases http://bigsdb.web.pasteur.fr/klebsiella/ klebsiella.html and https://pubmlst.org/bigsdb?db=pubmlst_abaumannii_pasteur_seqdef. A total of 11 different pulsotypes were identified among the K. pneumoniae collection, whilst 12 pulsotypes were detected among the Acinetobacter collection (Table 1). The six colistin-resistant K. pneumoniae isolates carried both the $b a_{\mathrm{OXA}-48}$ and $b l a_{\mathrm{NDM}-1}$ carbapenemase genes. MLST analysis revealed that these isolates belonged to ST101. Insertion of an insertion sequence (IS) identified as IS903B into the $\mathrm{mgrB}$ gene between nucleotides 107 and 108 for Kp65 and Kp82 isolates and insertion of an IS1R mobile element in the promotor region of the mgrB gene for Kp173, Kp174, Kp190 and Kp191 isolates (44 nucleotides before the GTG start codon) were detected by PCR amplification. These IS elements are likely to modify MgrB expression and to be responsible for the high-level colistin resistance observed in these isolates, as has been described previously [2]. PCR experiments did not reveal the presence of the mcr-like plasmid-mediated colistin resistance genes.

Monitoring the occurrence of multidrug-resistant bacteria is crucial to avoid the spread of such isolates in clinical facilities. Little information is known about the dissemination of carbapenemase-producers in Libya and only a few clinical cases of OXA-48-producing isolates recovered from Libyan refugees in Europe have been described [3,4]. However, more recently, an epidemiological study described a collection of NDM-1 and OXA23 carbapenemase-producing A. baumannii in a Libyan hospital [5]. Here we described multiresistant $K$. pneumoniae and A. baumannii isolates. To the best of our knowledge, this is the first study describing colistin-resistant and 16S RNA methylase-producing isolates in that area. This study underlines that multidrug resistance, possibly leading to pandrug resistance, may be spreading under-recognised in that part of the world. 
Table 1

Genotypic and phenotypic features of the collection of imipenem-non-susceptible Acinetobacter baumannii and Klebsiella spp. isolates.

\begin{tabular}{|c|c|c|c|c|c|c|c|}
\hline \multirow[t]{2}{*}{ Strain ID } & \multirow[t]{2}{*}{ Species } & \multirow[t]{2}{*}{ Pulsotype (MLST) } & \multirow[t]{2}{*}{ Sample } & \multicolumn{2}{|c|}{$\operatorname{MIC}(\mu \mathrm{g} / \mathrm{mL})$} & \multirow[t]{2}{*}{ Resistance gene(s) } & \multirow[t]{2}{*}{ Resistance phenotype } \\
\hline & & & & IPM & $\mathrm{COL}$ & & \\
\hline A37 & $A b$ & $10(\mathrm{ST} 2)$ & Sputum & $>32$ & 128 & $\operatorname{armA}$, bla $a_{\mathrm{OXA}-23}$ & AMK/CHL/COL/CIP/GEN/IPM/SUL/SXT/TET/TOB \\
\hline A29 & $A b$ & $1(\mathrm{ST} 164)$ & CSF & $>32$ & 32 & $b l a_{\mathrm{OXA}-23}$ & $\mathrm{CHL} / \mathrm{COL} / \mathrm{CIP} / \mathrm{GEN} / \mathrm{IPM} / \mathrm{TET} / \mathrm{TOB}$ \\
\hline A11 & $A b$ & $3(\mathrm{ST745)}$ & Eye & $>32$ & 8 & $b l a_{\mathrm{OXA}-23}$ & $\mathrm{CHL} / \mathrm{COL} / \mathrm{CIP} / \mathrm{GEN} / \mathrm{IPM} / \mathrm{SUL} / \mathrm{SXT} / \mathrm{TET} / \mathrm{TOB}$ \\
\hline A13 & $A b$ & 4 (ND) & Pleural fluid & $>32$ & 4 & $b^{b l a} a_{\mathrm{OXA}-23}$ & $\mathrm{CHL} / \mathrm{COL} / \mathrm{CIP} / \mathrm{GEN} / \mathrm{IPM} / \mathrm{SUL} / \mathrm{SXT} / \mathrm{TET} / \mathrm{TOB}$ \\
\hline A28 & $A b$ & $11(\mathrm{ST} 164)$ & Sputum & $>32$ & 4 & $b a_{\mathrm{OXA}-23}$ & $\mathrm{CHL} / \mathrm{COL} / \mathrm{CIP} / \mathrm{GEN} / \mathrm{IPM} / \mathrm{SUL} / \mathrm{SXT} / \mathrm{TET} / \mathrm{TOB}$ \\
\hline A31 & $A b$ & $3(\mathrm{ST745)}$ & ETT & $>32$ & 1 & $\operatorname{armA}, b l a_{\mathrm{OXA}-23}$ & AMK/CHL/CIP/GEN/IPM/SUL/SXT/TET/TOB \\
\hline A33 & $A b$ & 6 (ST2) & Blood & $>32$ & 2 & $\operatorname{armA}, b l a_{\mathrm{OXA}-23}$ & AMK/CHL/CIP/GEN/IPM/SUL/SXT/TET/TOB \\
\hline A34 & $A b$ & $8(\mathrm{ST} 2)$ & Urine & $>32$ & 0.5 & $\operatorname{armA}, b l a_{\mathrm{OXA}-23}$ & AMK/CHL/CIP/GEN/IPM/SUL/SXT/TET/TOB \\
\hline A36 & $A b$ & 9 (ST2) & Central line & $>32$ & 1 & $\operatorname{armA}$, bla $_{\mathrm{OXA}-23}$ & AMK/CHL/CIP/GEN/IPM/SUL/SXT/TET/TOB \\
\hline A76 & $A b$ & $8(\mathrm{ST} 2)$ & Urine & $>32$ & 2 & $\operatorname{armA}, b l a_{\mathrm{OXA}-23}$ & AMK/CHL/CIP/GEN/IPM/SUL/SXT/TET/TOB \\
\hline A87 & $A b$ & $6(\mathrm{ST} 2)$ & Ear & $>32$ & 0.5 & $\operatorname{armA}, b l a_{\mathrm{OXA}-23}$ & AMK/CHL/CIP/GEN/IPM/SUL/SXT/TET/TOB \\
\hline A19-a & $A b$ & $5(\mathrm{ST} 2)$ & Urine & $>32$ & 0.25 & $\operatorname{armA}, b l a_{\mathrm{OXA}-23}$ & AMK/CHL/CIP/GEN/IPM/SUL/SXT/TET/TOB \\
\hline A91 & $A b$ & 9 (ST2) & Swab & $>32$ & 0.5 & $\operatorname{arm} A, b l a_{\mathrm{OXA}-23}$ & AMK/CHL/CIP/GEN/IPM/SUL/SXT/TET/TOB \\
\hline A92 & $A b$ & 9 (ST2) & Sputum & $>32$ & 0.25 & $\operatorname{armA}, b l a_{\mathrm{OXA}-23}$ & AMK/CHL/CIP/GEN/IPM/SUL/SXT/TET/TOB \\
\hline A23 & $A b$ & 5 (ST2) & Blood & $>32$ & 1 & $b l a_{\mathrm{OXA}-23}$ & CHL/CIP/GEN/IPM/SUL/SXT/TET/TOB \\
\hline A20-a & $A b$ & $5(\mathrm{ST} 2)$ & Wound & $>32$ & 1 & $\operatorname{armA}$, bla $_{\text {OXА- } 23}$ & AMK/CHL/CIP/GEN/IPM/SUL/SXT/TET/TOB \\
\hline A32 & $A b$ & 7 (new ST) & Blood & $>32$ & 1 & $b l a_{\text {OXA-23 }}$ & CHL/CIP/GEN/IPM/SUL/SXT/TET/TOB \\
\hline A7 & $A b$ & $1(\mathrm{ST} 164)$ & Blood & $>32$ & 0.5 & $b^{b l a} a_{\mathrm{OXA}-23}$ & AMK/CHL/CIP/GEN/IPM/TET/TOB \\
\hline A15 & $A b$ & $3(\mathrm{ST7} 45)$ & Eye & $>32$ & 0.5 & bla $a_{\mathrm{OXA}-23}$ & AMK/CHL/CIP/IPM/SUL/SXT/TET/TOB \\
\hline A24 & $A b$ & $6(\mathrm{ST} 2)$ & Blood & $>32$ & 0.5 & $b l a_{\mathrm{OXA}-23}$ & AMK/CHL/CIP/GEN/IPM/SUL/SXT/TET/TOB \\
\hline A27 & $A b$ & $2(\mathrm{ST} 164)$ & ETT & $>32$ & 0.5 & $b l a_{\mathrm{OXA}-23}$ & AMK/CHL/CIP/GEN/IPM/TET/TOB \\
\hline A86 & $A b$ & $6(\mathrm{ST} 2)$ & Central line & $>32$ & 0.5 & bla $a_{\mathrm{OXA}-23}$ & AMK/CHL/CIP/GEN/IPM/SUL/SXT/TET/TOB \\
\hline A8 & $A b$ & 1 (ST164) & ETT & $>32$ & 0.25 & bla $a_{\mathrm{OXA}-23}$ & AMK/CHL/CIP/GEN/IPM/SUL/SXT/TET/TOB \\
\hline A17 & $A b$ & $2(\mathrm{ST} 164)$ & UVC & $>32$ & 0.25 & bla $_{\mathrm{OXA}-23}$ & CHL/CIP/GEN/IPM/SUL/TET/TOB \\
\hline A35 & $A b$ & 1 (ST164) & CSF & $>32$ & 0.25 & $\operatorname{arm} A$, bla $a_{\mathrm{OXA}-23}$ & AMK/CIP/GEN/IPM/SUL/SXT/TET/TOB \\
\hline A39 & $A b$ & $12(\mathrm{ST} 164)$ & Swab & $>32$ & 0.25 & $b l a_{\mathrm{OXA}-23}$ & AMK/CHL/CIP/GEN/IPM/TET/TOB \\
\hline A10 & $A b$ & $2(\mathrm{ST} 164)$ & UVC & $>32$ & 0.125 & $b l a_{\mathrm{OXA}-23}$ & AMK/CHL/CIP/GEN/IPM/TET/TOB \\
\hline A16 & $A b$ & $2(\mathrm{ST} 164)$ & UVC & $>32$ & 0.125 & $b l a_{\mathrm{OXA}-23}$ & AMK/CIP/GEN/IPM/SUL/TET/TOB \\
\hline Кр173 & $K p$ & $1(\mathrm{ST} 101)$ & Pus & 16 & 128 & $b l a_{\mathrm{OXA}-48}, b l a_{\mathrm{NDM}-1}$ & CIP/COL/GEN/IPM/SUL/SXT/TET/TOB \\
\hline Kp174 & $K p$ & 1 (ST101) & Blood & 16 & 64 & $b l a_{\mathrm{OXA}-48}, b l a_{\mathrm{NDM}-1}$ & $\mathrm{CIP} / \mathrm{COL} / \mathrm{GEN} / \mathrm{IPM} / \mathrm{SUL} / \mathrm{SXT} / \mathrm{TET} / \mathrm{TOB}$ \\
\hline Kp190 & $K p$ & 1 (ST101) & Blood & 16 & 128 & $b l a_{\mathrm{OXA}-48}, b l a_{\mathrm{NDM}-1}$ & CIP/COL/GEN/IPM/SUL/TET/TOB \\
\hline Kp191 & $K p$ & 1 (ST101) & Sputum & 16 & 128 & $b l a_{\mathrm{OXA}-48}, b l a_{\mathrm{NDM}-1}$ & CIP/COL/GEN/IPM/SUL/TET/TOB \\
\hline Kp65 & $K p$ & 2 (ST101) & Urine & 2 & 64 & $b l a_{\mathrm{OXA}-48}, b l a_{\mathrm{NDM}-1}$ & AMK/CIP/COL/GEN/IPM/SXT/TET/TOB \\
\hline Kp82 & $K p$ & $2(\mathrm{ST} 101)$ & Swab & 3 & 64 & $b l a_{\mathrm{OXA}-48}, b l a_{\mathrm{NDM}-1}$ & AMK/CIP/COL/GEN/IPM/SXT/TET/TOB \\
\hline Kp50 & $K p$ & $11(\mathrm{ST} 15)$ & Sputum & 3 & 0.125 & bla $_{\mathrm{OXA}-48}$, bla $_{\mathrm{NDM}-1}, r m t C$ & AMK/CIP/GEN/IPM/SUL/SXT/TOB \\
\hline Kp69 & Ko & $9(\mathrm{ND})$ & Central line & 8 & 0.125 & $b l a_{\mathrm{OXA}-48}, b l a_{\mathrm{NDM}-1}, r m t C$ & AMK/GEN/IPM/SUL/TOB \\
\hline Kp93 & $K p$ & $3(\mathrm{ST} 101)$ & ETT & 3 & 0.125 & $b l a_{\mathrm{OXA}-48}, b l a_{\mathrm{NDM}-1}, r m t C$ & AMK/CIP/GEN/IPM/SUL/SXT/TET/TOB \\
\hline Kр42 & $K p$ & 8 (ST147) & Wound & 1 & 0.125 & $b l a_{\mathrm{OXA}-48}, b l a_{\mathrm{NDM}-1}$ & CHL/CIP/GEN/IPM/TET/TOB \\
\hline Kp51 & $K p$ & 11 (ST15) & Urine & $>32$ & 0.125 & bla $_{\mathrm{OXA}-48}$, bla $_{\mathrm{NDM}-1}, r m t C$ & AMK/CIP/GEN/IPM/SUL/SXT/TOB \\
\hline Kp53 & $K p$ & 7 (ST147) & Swab & 1 & 0.125 & $b l a_{\mathrm{OXA}-48}, b l a_{\mathrm{NDM}-1}$ & CIP/GEN/IPM/SUL/SXT/TET/TOB \\
\hline Kp58 & $K p$ & 7 (ST147) & Urine & 1 & 0.125 & $b l a_{\mathrm{OXA}-48}, b l a_{\mathrm{NDM}-1}$ & CIP/GEN/IPM//TET/TOB \\
\hline Kp61 & $K p$ & $10(\mathrm{ST} 11)$ & ETT & $>32$ & 0.125 & $b l a_{\mathrm{OXA}-48}, b l a_{\mathrm{NDM}-1}$ & AMK/CHL/CIP/GEN/IPM/SUL/SXT/TET/TOB \\
\hline Kp52 & $K p$ & 7 (ST147) & Blood & 1 & 0.125 & $b l a_{\mathrm{OXA}-48}, b l a_{\mathrm{NDM}-1}$ & CIP/GEN/SUL/SXT/TET/TOB \\
\hline Kp59 & $K p$ & $10(\mathrm{ST} 11)$ & Catheter & $>32$ & 0.125 & $b^{\prime} a_{\mathrm{NDM}-1}$ & AMK/CHL/CIP/GEN/IPM/SUL/SXT/TOB \\
\hline Kp60 & $K p$ & 10 (ST11) & Swab & $>32$ & 0.125 & $b l a_{\mathrm{NDM}-1}$ & AMK/CIP/GEN/IPM/SUL/SXT/TOB \\
\hline Kp78 & $K p$ & $2(\mathrm{ST} 101)$ & Urine & $>32$ & 0.125 & $b l a_{\mathrm{NDM}-1}$ & CIP/GEN/IPM/SUL/TET/TOB \\
\hline Kp94 & $K p$ & 7 (ST147) & Nose & 0.5 & 0.125 & $b_{\text {NDM-1 }}$ & CIP/GEN/SUL/SXT/TET/TOB \\
\hline Kp12 & $K p$ & 5 (ST405) & Wound & 1 & 0.125 & bla $a_{\mathrm{NDM}-1}$ & CIP/GEN/IPM/SUL/SXT/TET/TOB \\
\hline Kp25 & $K p$ & 2 (ST101) & Wound & 3 & 0.125 & $b l a_{\mathrm{OXA}-48}$ & CIP/GEN/IPM/SXT/TET/TOB \\
\hline Kp88 & $K p$ & 5 (ST405) & Blood & 0.75 & 0.125 & bla $a_{\mathrm{OXA}-48}$ & CIP/GEN/SUL/SXT/TET/TOB \\
\hline Kp89 & $K p$ & 5 (ST405) & ETT & 0.75 & 0.125 & $b l a_{\mathrm{OXA}-48}$ & CIP/GEN/SUL/SXT/TET/TOB \\
\hline Kp84 & $K p$ & $2(\mathrm{ST} 101)$ & ETT & 0.38 & 0.125 & $b l a_{\mathrm{OXA}-48}$ & CIP/GEN/SUL/SXT/TET/TOB \\
\hline Kp95 & $K p$ & 3 (ST101) & ETT & 0.38 & 0.125 & $b l a_{\mathrm{OXA}-48}$ & CIP/GEN/TET/TOB \\
\hline Kр49 & $K p$ & 8 (ST147) & Sputum & 0.5 & 0.125 & bla $a_{\mathrm{OXA}-48}$ & AMK/CIP/GEN/SUL/SXT/TET/TOB \\
\hline Kp56 & $K p$ & 6 (ST405) & ETT & 0.5 & 0.125 & bla $a_{\mathrm{OXA}-48}$ & CIP/GEN/SUL/SXT/TET/TOB \\
\hline Kp57 & $K p$ & 6 (ST405) & Blood & 0.5 & 0.125 & bla $a_{\mathrm{OXA}-48}$ & CIP/GEN/SUL/SXT/TET/TOB \\
\hline Kp70 & $K p$ & 6 (ST405) & Blood & 0.5 & 0.125 & $b l a_{\mathrm{OXA}-48}$ & CIP/GEN/SUL/SXT/TET/TOB \\
\hline Kp72 & $K p$ & 5 (ST405) & ETT & 0.5 & 0.125 & bla $a_{\mathrm{OXA}-48}$ & CIP/GEN/SUL/SXT/TET/TOB \\
\hline Kp80 & $K p$ & 4 (ST45) & ETT & 0.5 & 0.125 & bla $a_{\mathrm{OXA}-48}$ & GEN/SXT/TET/TOB \\
\hline Kp71 & $K p$ & $2(\mathrm{ST} 101)$ & Blood & 0.5 & 0.125 & $b l a_{\mathrm{OXA}-48}$ & CIP/GEN/TET/TOB \\
\hline Kp85 & $K p$ & 3 (ST101) & ETT & 0.5 & 0.125 & $b l a_{\mathrm{OXA}-48}$ & CIP/GEN/SUL/SXT/TET/TOB \\
\hline
\end{tabular}

Ab, Acinetobacter baumannii; Kp, Klebsiella pneumoniae; Ko, Klebsiella oxytoca; MLST, multilocus sequence typing; ND, not determined; CSF, cerebrospinal fluid; ETT, endotracheal tube; UVC, umbilical vein catheter; MIC, minimum inhibitory concentration; IPM, imipenem; COL, colistin; AMK, amikacin; CHL, chloramphenicol; CIP, ciprofloxacin; GEN, gentamicin; SUL, sulfonamide; SXT, trimethoprim/sulfamethoxazole; TET, tetracycline; TOB, tobramycin. 


\section{Funding}

This work was funded by the University of Fribourg (Fribourg, Switzerland).

\section{Competing interests}

None declared.

\section{Ethical approval}

Not required.

\section{References}

[1] Nordmann P, Jayol A, Poirel L. Rapid detection of polymyxin resistance in Enterobacteriaceae. Emerg Infect Dis 2016;22:1038-43.

[2] Poirel L, Jayol A, Nordmann P. Polymyxins: antibacterial activity, susceptibility testing, and resistance mechanisms encoded by plasmids or chromosomes. Clin Microbiol Rev 2017:30:557-96.

[3] Pirš M, Andlovic A, Cerar T, Žohar-Čretnik T, Kobola L, Kolman J, et al. A case of OXA-48 carbapenemase-producing Klebsiella pneumoniae in a patient transferred to Slovenia from Libya. Euro Surveill 2011;16:20042.

[4] Kocsis E, Savio C, Piccoli M, Cornaglia G, Mazzariol A. Klebsiella pneumoniae harbouring OXA-48 carbapenemase in a Libyan refugee in Italy. Clin Microbiol Infect 2013;19:E409-11.

[5] Mathlouthi N, El Salabi AA, Ben Jomàa-Jemili M, Bakour S, Al-Bayssari C, Zorgani $A A$, et al. Early detection of metallo- $\beta$-lactamase NDM- 1 and OXA-23 carbapenemase-producing Acinetobacter baumannii in Libyan hospitals. Int J Antimicrob Agents 2016;48:46-50.

Nicolas Kieffer ${ }^{\mathrm{a}, \mathrm{b}, \mathrm{c}}$

${ }^{a}$ 'Emerging Antibiotic Resistance', Medical and Molecular Microbiology Unit, Department of Medicine, Faculty of Science, University of Fribourg, Fribourg, Switzerland

${ }^{b}$ INSERM European Unit (LEA Paris), University of Fribourg, Fribourg, Switzerland

${ }^{c}$ Swiss National Reference Center for Emerging Antibiotic Resistance (NARA), Fribourg, Switzerland

Mohamed O. Ahmed Faculty of Veterinary Medicine, Department of Microbiology and Parasitology, University of Tripoli, Tripoli, Libya
Asma K. Elramalli Tripoli Medical Centre, Tripoli, Libya

Mohamed A. Daw Department of Medical Microbiology, Faculty of Medicine, University of Tripoli, Tripoli, Libya

Laurent Poirel ${ }^{\mathrm{a}, \mathrm{b}, \mathrm{c}}$

${ }^{a}$ 'Emerging Antibiotic Resistance', Medical and Molecular Microbiology Unit, Department of Medicine, Faculty of Science, University of Fribourg, Fribourg, Switzerland

${ }^{b}$ INSERM European Unit (LEA Paris), University of Fribourg, Fribourg, Switzerland

${ }^{c}$ Swiss National Reference Center for Emerging Antibiotic Resistance (NARA), Fribourg, Switzerland

Rocío Álvarez

Unit of Infectious Diseases, Microbiology and Preventive Medicine and Biomedical Institute of Seville (IBiS), University Hospital Virgen del Rocio/CSIC/University of Seville, Seville, Spain

Patrice Nordmann ${ }^{\mathrm{a}, \mathrm{b}, \mathrm{c}, \mathrm{d}, *}$

${ }^{a}$ 'Emerging Antibiotic Resistance', Medical and Molecular Microbiology Unit, Department of Medicine, Faculty of Science, University of Fribourg, Fribourg, Switzerland

${ }^{b}$ INSERM European Unit (LEA Paris), University of Fribourg, Fribourg, Switzerland

${ }^{c}$ Swiss National Reference Center for Emerging Antibiotic Resistance (NARA), Fribourg, Switzerland

${ }^{d}$ Institute for Microbiology, University of Lausanne and University Hospital Centre, Lausanne, Switzerland

* Corresponding author at: 'Emerging Antibiotic Resistance', Medical and Molecular Microbiology Unit, Department of Medicine, Faculty of Science, University of Fribourg, Chemin du Musée 18, Fribourg, Switzerland. 